MATHEMATICS OF COMPUTATION

Volume 78, Number 266, April 2009, Pages 1119-1125

S 0025-5718(08)02120-0

Article electronically published on September 8, 2008

\title{
TRACE OF TOTALLY POSITIVE ALGEBRAIC INTEGERS AND INTEGER TRANSFINITE DIAMETER
}

\author{
V. FLAMMANG
}

\begin{abstract}
Explicit auxiliary functions can be used in the "Schur-SiegelSmyth trace problem". In the previous works, these functions were constructed only with polynomials having all their roots positive. Here, we use several polynomials with complex roots, which are found with Wu's algorithm, and we improve the known lower bounds for the absolute trace of totally positive algebraic integers. This improvement has a consequence for the search of Salem numbers that have a negative trace. The same method also gives a small improvement of the upper bound for the integer transfinite diameter of $[0,1]$.
\end{abstract}

\section{INTRODUCTION}

1.1. The trace of totally positive algebraic integers. Let $\alpha$ be a totally positive algebraic integer of degree $d \geq 2$ (i.e. its conjugates $\alpha_{1}=\alpha, \ldots, \alpha_{d}$ are all positive real numbers) and let $P$ be its minimal polynomial. We define the absolute trace of $\alpha$ as

$$
\operatorname{Trace}(\alpha)=\frac{1}{d} \operatorname{trace}(\alpha)=\frac{1}{d} \sum_{i=1}^{d} \alpha_{i}
$$

and denote by $\mathcal{T}$ the set of all such $\operatorname{Trace}(\alpha)$.

The "Schur-Siegel-Smyth trace problem" (so called by P. Borwein in his book [B] ) is the following: Fix $\rho<2$. Then show that all but finitely many totally positive algebraic integers $\alpha$ have $\operatorname{Trace}(\alpha)>\rho$.

Remark. Solving this problem is equivalent to proving that 2 is the smallest limit point of $\mathcal{T}$.

The problem was solved in 1918 by I. Schur for $\rho<\sqrt{e}[\underline{\mathrm{Sc}}$; in 1943 by C. L. Siegel for $\rho<1.7337$ [Si] and in 1984 by C. J. Smyth for $\rho<1.7719$ [Sm2]. More recently, it was solved in 2004 by J. F. McKee and C. J. Smyth for $\rho<1.7783786$ [MS], and in 2006 by J. Aguirre and J. C. Peral for $\rho<1.784109$ [AP]. The method of proof, as in [Sm1], uses an explicit auxiliary function of the following type:

$$
\text { for } x>0, f(x)=x-\sum_{1 \leq j \leq J} c_{j} \log \left|Q_{j}(x)\right| \geq m,
$$

Received by the editor April 2, 2007 and, in revised form, June 22, 2007.

2000 Mathematics Subject Classification. Primary 11R04, 11Y40.

(C)2008 American Mathematical Society 
where the $c_{j}$ are positive real numbers and the polynomials $Q_{j}$ are nonzero elements of $\mathbb{Z}[X]$. Then

$$
\sum_{i=1}^{d} f\left(\alpha_{i}\right) \geq m d
$$

i.e.

$$
\operatorname{trace}(\alpha) \geq m d+\sum_{1 \leq j \leq J} c_{j} \log \left|\prod_{i=1}^{d} Q_{j}\left(\alpha_{i}\right)\right| .
$$

We assume that $P$ does not divide any $Q_{j}$, then $\prod_{i=1}^{d} Q_{j}\left(\alpha_{i}\right)$ is a nonzero integer because it is the resultant of $P$ and $Q_{j}$. Therefore, if $\alpha$ is not a root of $Q_{j}$, we have

$$
\operatorname{Trace}(\alpha) \geq m \text {. }
$$

On the other hand, J. P. Serre (see Appendix B in [AP]) showed that this method does not give such an inequality for any $\rho$ larger than $1.8983021 \ldots$. Therefore, this method cannot be used to prove that 2 is the smallest limit point of $\mathcal{T}$. Nevertheless, it is interesting to try to get lower bounds for $\operatorname{Trace}(\alpha)$. For instance, this was used for the search of Salem numbers of smallest degree with trace equal to -2 by J. F. McKee and C. J. Smyth MS. We will explain in Section 1.2 the consequence of our bound for the degree of Salem numbers of trace -3 .

In this paper, we prove the following:

Theorem 1. If $\alpha$ is a totally positive algebraic integer, then, with a finite set of explicit exceptions (see Table 11), we have

$$
\frac{1}{d} \operatorname{trace}(\alpha) \geq 1.78702 \text {. }
$$

The main point is to find a good list of polynomials $Q_{j}$ which gives a value of $m$ as large as possible. In [Sm2] and [AP] the authors used an extended heuristic search to get polynomials of the following type: all their roots are positive and they have small absolute trace. Here we use a new approach relying on the auxiliary function (1.1) to a generalization of the integer transfinite diameter. Our polynomials are found by Wu's algorithm $[\mathrm{Wu}$. Surprisingly we get a lot of polynomials with some complex roots. This phenomenon has already been encountered by Habsieger and Salvy [HS] when they were studying the integer transfinite diameter of $[0,1]$. Their exceptional polynomial (after a natural transformation) will appear during our search but will not be used in the final result (1.2). The complete list of polynomials $Q_{j}$ and coefficients $c_{j}$ is given in Table 2 ,

1.2. Salem numbers of trace -3. A Salem number is a real algebraic integer greater than 1 whose conjugates all lie in the closed disc $|z| \leq 1$, with at least one on the unit circle. Its minimal polynomial is a reciprocal polynomial of degree $2 d \geq 4$. Finding all Salem numbers of degree $2 d$ and trace -3 is equivalent to finding all totally positive algebraic integers $\alpha$ of degree $d$ and trace $2 d-3$ such that $\alpha>4$ and all other conjugates of $\alpha$ are in the interval ]0,4[. In fact, let

$$
P(x)=x^{d}-(2 d-3) x^{d-1}+\ldots
$$

be the minimal polynomial of such a totally positive algebraic integer. The transformation $x=z+\frac{1}{z}+2$ produces a reciprocal polynomial

$$
Q(z)=z^{2 d}+3 z^{2 d-1}+\cdots+3 z+1
$$


which is the minimal polynomial of a Salem number of degree $2 d$ and trace -3 because the roots of $P$ in the interval ]0,4[ give pairs of roots of $Q$ on the unit circle while the root of $P$ in the interval $] 4,+\infty[$ gives a pair of reciprocal real positive roots of $Q$. We prove the following:

Theorem 2. If a Salem number has trace -3, then its degree is at least 30 .

It is an easy consequence of Theorem 1 . As $1.78702>\frac{25}{14}$, then there exists no totally positive irreducible polynomial of degree 14 and trace 25 (corresponding or not to a Salem number of trace -3). Thus, the next largest possible degree for such a polynomial is 15 and so at least degree 30 for the corresponding Salem number. The previous bound for the absolute trace of a totally positive algebraic integer was 1.784109 [AP]. This proved that the minimal degree for a Salem number of trace -3 was at least 28 .

1.3. The integer transfinite diameter. For a polynomial $P \in \mathbb{Z}[X]$ and a real interval $I=[a, b]$ we denote $|P|_{\infty, I}=\sup _{z \in I}|P(z)|$. We define the integer transfinite diameter of $I$ by

$$
t_{\mathbb{Z}}(I)=\liminf _{\substack{n \geq 1 \\ n \rightarrow+\infty}} \inf _{\substack{P \in \mathbb{Z}[X] \\ \operatorname{deg}(P)=n}}|P|_{\infty, I}^{\frac{1}{n}}
$$

Many authors have given lower and upper bounds of $t_{\mathbb{Z}}(I)$, especially when $I=[0,1]$ (see $[\mathrm{AP}]$ and $\left[\mathrm{Pr}\right.$ for an account of this). It is known that $t_{\mathbb{Z}}([0,1])=$ $t_{\mathbb{Z}}([0,1 / 4])^{1 / 2}$. To get an upper bound for $t_{\mathbb{Z}}([0,1 / 4])$, it is sufficient to get an explicit polynomial $Q \in \mathbb{Z}[X]$ and then to use the sequence of the successive powers of $Q$. So we search a polynomial $Q$ of degree $r$ such that $|Q|_{\infty, I}^{1 / r} \leq e^{-m}$. By the change of variable $x \mapsto \frac{1}{x+4}$ and taking the logarithm we get

$$
\text { for } x>0, f(x)=\log (x+4)-\sum_{1 \leq j \leq J} c_{j} \log \left|Q_{j}(x)\right| \geq m,
$$

where the numbers $c_{j}$ are positive rational numbers satisfying the condition $\sum_{1 \leq j \leq J} c_{j} \operatorname{deg} Q_{j} \leq 1$ and the polynomials $Q_{j}$ are obtained from the irreducible factors of $Q$ by the transformation above. It is plain that the algorithm given in Section 2 for the auxiliary function (1.1) is also convenient for the function (1.4). We get

Result 1.

$$
t_{\mathbb{Z}}([0,1])<0.42291334 .
$$

This is a small improvement on the upper bound 0.42305209 given by Aguirre and Peral $\mathrm{AP}$. The best known lower bound 0.4213 was given by Pritsker $[\mathrm{Pr}]$. Here we also have several factors of $Q$, including the polynomial of Habsieger and Salvy, which has some complex roots.

In Section 2 we explain how to construct the auxiliary function (1.1) (the same method is used to construct the function (1.4)). The numerical results are given in Section 3. All the computations are done on an iBook Macintosh with the languages Pascal, Maple and Pari. 


\section{Construction of the EXPlicit AUXiliary FUnCtion}

2.1. Rewriting the auxiliary function. Inside the auxiliary function (1.1) we replace the numbers $c_{j}$ by rational numbers.

So, we may write:

$$
\text { for } x>0, f(x)=x-\frac{t}{r} \log |Q(x)| \geq m
$$

where $Q \in \mathbb{Z}[X]$ is of degree $r$ and $t$ is a positive real number. We want to get a function $f$ whose minimum $m$ on $(0, \infty)$ is as large as possible. Thus we search a polynomial $Q \in \mathbb{Z}[X]$ such that

$$
\sup _{x>0}|Q(x)|^{t / r} e^{-x} \leq e^{-m} .
$$

If we suppose that $t$ is fixed, we need to get an effective upper bound for the quantity

$$
t_{\mathbb{Z}, \varphi}([0, \infty))=\liminf _{\substack{r \geq 1 \\ r \rightarrow+\infty}} \inf _{\substack{P \in \mathbb{Z}[X] \\ \operatorname{deg}(P)=r}} \sup _{x>0}\left(|P(x)|^{\frac{t}{r}} \varphi(x)\right)
$$

in which we use the weight $\varphi(x)=e^{-x}$.

It is clear that this quantity is closely related to the usual integer transfinite diameter of an interval given by (1.3).

2.2. How to find the polynomials $Q_{j}$. We first take an initial value of $t$, say $t_{0}=1$ and a set $E_{0}$ of 50 control points uniformly distributed on $[0,2.5]$. With Wu's algorithm $[\mathrm{Wu}$, we compute a polynomial $Q$ of degree at most 10 which is small on $E_{0}$. We define $Q_{1}$ as the irreducible factor of $Q$ of smallest degree and we take the best value of $c_{1}$ to get the best auxiliary function $f_{1}$. We deduce from this the value $t_{1}=c_{1} \operatorname{deg}\left(Q_{1}\right)$. We add to the set $E_{0}$ the points of $[0,+\infty)$ where $f_{1}$ has a local minimum (including those greater than 2.5) to get a new set $E_{1}$ of control points. With Wu's algorithm we compute a polynomial $Q$ of degree $10+\operatorname{deg} Q_{1}$ which is a multiple of $Q_{1}$ of small norm on $E_{1}$ and take $Q_{2}$ as another irreducible factor of $Q$. We optimize $\left(c_{1}, c_{2}\right)$ to get the best function $f_{2}$. This gives $t_{2}$. We get the set $E_{2}$ from $E_{1}$ adding the local minima of $f_{2}$. Then we search a polynomial $Q$ which is a multiple of $Q_{1} Q_{2}$ of degree $10+\operatorname{deg} Q_{1}+\operatorname{deg} Q_{2}$ and we continue this process until two consecutive steps produce no new polynomial.

Remark. The previous algorithm is used repeatedly with the constant 10 replaced successively by 11 to 20 .

We give a numerical example of search for degree 13 .

We start with $E_{0}$ and $t_{0}$ defined as above. We search a polynomial $Q$ of degree 13 such that $x Q(x)$ is small on $E_{0}$. LLL produces the polynomial $Q(x)=$ $x^{7}(x-1)^{4}(2 x-1)(3 x-1)$. We optimize the auxiliary function

$$
f_{1}=x-c_{1} \log x-c_{2} \log |x-1|-c_{3} \log |2 x-1|-c_{4} \log |3 x-1| .
$$

Only $c_{1}$ and $c_{2}$ are nonzero. We deduce the value of $t_{1}=c_{1} \operatorname{deg}(x)+c_{2} \operatorname{deg}(x-1)=$ 1.606. We add to the set $E_{0}$ the two points where $f_{1}$ has a local minimum and we get a new set $E_{1}$ of control points. We repeat LLL and now search for a polynomial $Q$ such that $x^{8}(x-1)^{4} Q(x)$ is small on $E_{1}$. At the 8 th step, LLL produces the 
polynomial

$$
\begin{array}{r}
Q(x)=(x-1)\left(x^{2}-3 x+1\right)\left(x^{5}-9 x^{4}+27 x^{3}-31 x^{2}+12 x-1\right) \\
\underline{\left(x^{5}-8 x^{4}+22 x^{3}-25 x^{2}+10 x-1\right) .}
\end{array}
$$

The underlined polynomial is related to the exceptional polynomial (i.e. with some complex roots) obtained by Habsieger and Salvy as explained in Section 3. We test the two new polynomials in the auxiliary function. Both have nonzero exponents. At this point, we have $\frac{1}{d} \operatorname{trace}(\alpha) \geq 1.762$. The polynomials used in the auxiliary function are the polynomials $1,2,3,4,6,7,11,12,14$ in Table 1 and the underlined polynomial above.

2.3. Optimization of the $c_{j}$. For the optimization of the auxiliary function we use the semi-infinite linear programming method introduced into number theory by Smyth Sm1]. We recall it briefly. We define by induction a sequence of finite sets $X_{n}, n \geq 0$, with $X_{n} \subset[0,+\infty)$. We start with an arbitrary set of points $X_{0}$ of cardinal greater than $J$. At each step $n \geq 0$, we compute the best values for $c_{j}$ by linear programming on the set $X_{n}$. We get a function $f_{n}$ whose minimum $m_{n}=\min _{x \in X_{n}} f_{n}(x)$ is greater than $m_{n}^{\prime}=\min _{x>0} f_{n}(x)$. We add to $X_{n}$ the points of $[0,+\infty)$ where $f_{n}$ has a local minimum smaller than $m_{n}+\epsilon_{n}$, where $\left(\epsilon_{n}\right)_{n \geq 0}$ is a decreasing sequence of positive numbers tending to 0 when $n$ is increasing and chosen such that the set $X_{n}$ does not increase too quickly. We stop, for instance, when $m_{n}-m_{n}^{\prime}<10^{-6}$. If $k$ steps are necessary, we take $m=m_{k}^{\prime}$.

\section{Numerical Results}

The method described above gives, among others, the 35 polynomials listed in Table 1 and the 28 polynomials listed in Table 2 . Among these polynomials, 17 are new.

Four of them are minimal polynomials of totally positive algebraic integers.

The last thirteen polynomials have at least two complex roots. They are of the same type: their real parts all lie in $[0,5.2]$ and their imaginary parts are small, i.e $|\operatorname{Im}(z)|<0.6325$.

The first new polynomial of this family that appeared was $x^{5}-8 x^{4}+22 x^{3}-$ $25 x^{2}+10 x-1$. It has two complex roots, which has reminded us of the exceptional polynomial of Habsieger and Salvy. We transform this polynomial by $x \mapsto \frac{1}{x}-4$ in order to get a polynomial whose real roots are in $\left[0, \frac{1}{4}\right]$. We find the polynomial $4921 x^{5}-4594 x^{4}+1697 x^{3}-310 x^{2}+28 x-1$ which is the polynomial $A_{8}$ in [HS. But this polynomial does not belong to the final list of our polynomials. 
TABLE 1 . List of the polynomials $Q_{j}$ that occur in the explicit auxiliary function (1.1) to obtain the inequality (1.2). Those marked with a sharp are the exceptions mentioned in Theorem 1. Those marked with an asterisk are only used for the lower bound of $t_{\mathbb{Z}}([0,1]) . \quad \nu$ is the number of complex roots of the new polynomials $Q_{j}$.

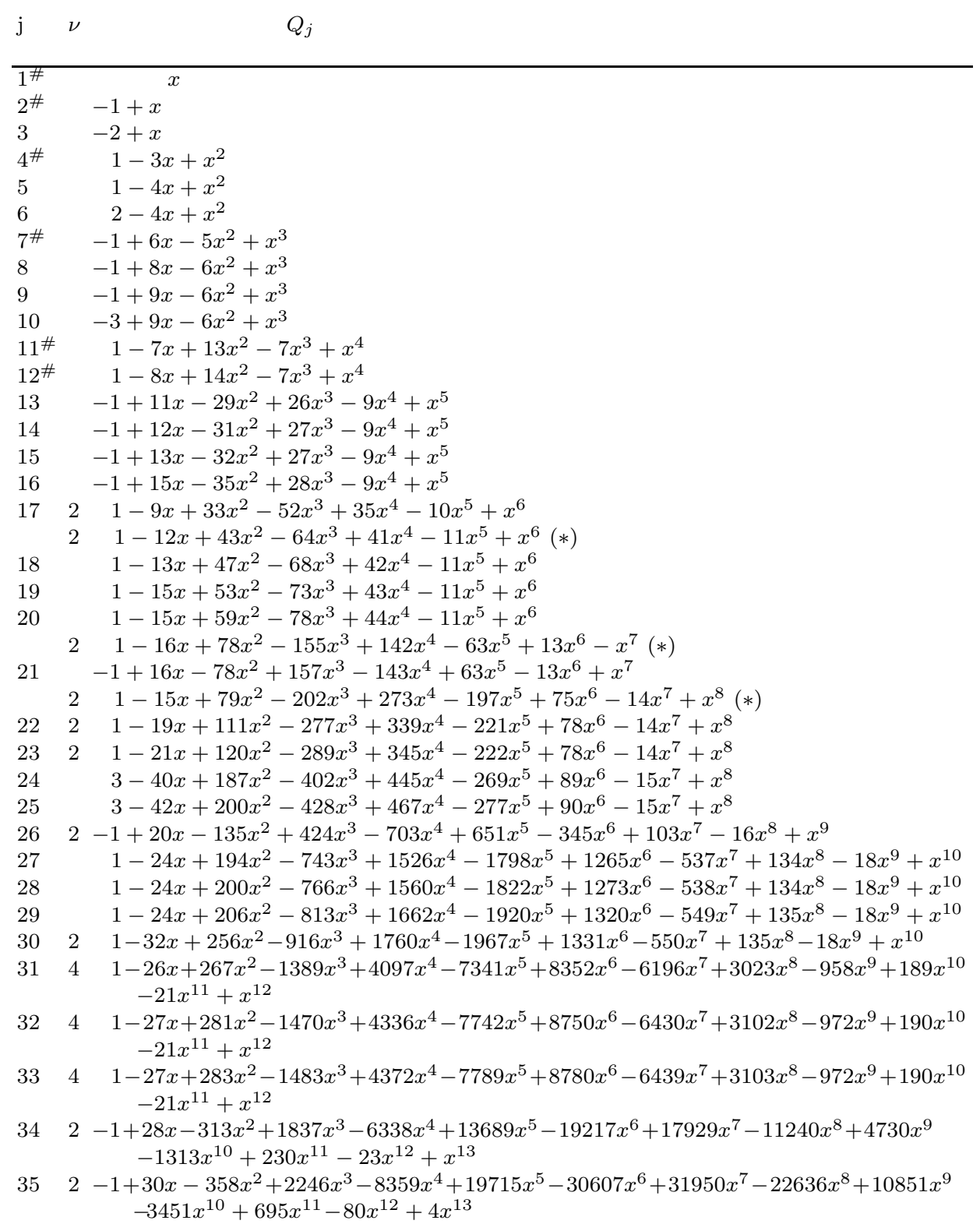


TABLE 2. List of the coefficients $c_{j}$ used in the explicit auxiliary function (1.1)

\begin{tabular}{lccccccc}
$j$ & $c_{j}$ & $j$ & $c_{j}$ & $j$ & $c_{j}$ & $j$ & $c_{j}$ \\
\hline & & & & & & & \\
\hline 1 & 0.5584957222 & 10 & 0.003856972798 & 19 & 0.001108761904 & 28 & 0.004813507609 \\
2 & 0.4916967610 & 11 & 0.02993703111 & 20 & 0.0008466162920 & 29 & 0.004620732100 \\
3 & 0.07630565169 & 12 & 0.02755084495 & 21 & 0.0008291782706 & 30 & 0.003043369627 \\
4 & 0.1761207656 & 13 & 0.005156588163 & 22 & 0.001195618507 & 31 & 0.001938859038 \\
5 & 0.01072483141 & 14 & 0.007670086583 & 23 & 0.002200027138 & 32 & 0.003180059759 \\
6 & 0.01038787553 & 15 & 0.003542904754 & 24 & 0.001352021285 & 33 & 0.006514951360 \\
7 & 0.07277644358 & 16 & 0.004673480798 & 25 & 0.004580671504 & 34 & 0.002183415705 \\
8 & 0.002809414882 & 17 & 0.001557114885 & 26 & 0.0008445959086 & 35 & 0.001482155949 \\
9 & 0.005946815966 & 18 & 0.003429925309 & 27 & 0.004268551700 & &
\end{tabular}

\section{ACKNowledgement}

This work would not have been possible without the precious help of Professor Georges Rhin in the computation programs. I am very much indebted to him.

\section{REFERENCES}

[AP] J. Aguirre and J.C. Peral. The trace problem for totally positive algebraic integers, Number Theory and Polynomials. (Conference proceedings, University of Bristol, 3-7 April 2006, editors J.F. McKee and C.J. Smyth). LMS Lecture notes.

[B] P. Borwein. Computational excursions in analysis and number theory, CMS Books in Mathematics, 10. Springer-Verlag, New York, 2002. MR1912495 (2003m:11045)

[F] V. Flammang. Sur le diamètre transfini entier d'un intervalle à extrémités rationnelles, Ann. Inst. Fourier, Grenoble 45 (1995), 779-793. MR1340952 (96i:11083)

[HS] L. Habsieger and B. Salvy. On integer Chebyshev polynomials, Math. Comp. 218 (1997), 763-770. MR 1401941 (97f:11053)

[MS] J.F. McKee and C.J. Smyth. Salem numbers of trace -2 and traces of totally positive algebraic integers, ANTS 2004, LNCS 3076, (2004), 327-337. MR2137365 (2006a:11134)

[Pr] I.E. Pritsker Small polynomials with integer coefficients, J. Anal. Math. 96 (2005), 151-190. MR 2177184 (2006j:11033)

[Sc] I. Schur. Uber die Verteilung der Wurzeln bei gewissen algebraischen Gleichungen mit ganzzahligen Koeffizienten, Math. Z. 1 (1918), 377-402. MR.1544303

[Si] C.L. Siegel. The trace of totally positive and real algebraic integers, Ann. of Maths, 46 (1945), 302-312. MR0012092 (6:257a)

[Sm1] C.J. Smyth. The mean value of totally real algebraic numbers, Math. Comp. 42 (1984) 663-681. MR736460 (86e:11115)

[Sm2] C.J. Smyth. Totally positive algebraic integers of small trace, Ann. Inst. Fourier, Grenoble 33 (1984), 1-28. MR762691 (86f:11091)

[Sm3] C.J. Smyth. An inequality for polynomials, Number theory (Ottawa, ON, 1996), 315-321, CRM Proc. Lecture Notes, 19, Amer. Math. Soc., Providence, RI, 1999. MR1684612 (2000d:11145)

[Wu] Q. Wu. On the linear independence measure of logarithms of rational numbers, Math. Comp. 72 (2003), 901-911 MR1954974 (2003m:11111)

UMR CNRS 7122 Département de Mathématiques, UFR MiM, Université Paul Verlaine de Metz, Ile du Saulcy, 57045 MetZ cedex 01, France

E-mail address: flammang@univ-metz.fr 